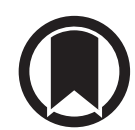

CrossMark

\title{
Use of Xpert MTB/RIF Ultra assays among paediatric tuberculosis experts in Europe
}

\author{
To the Editor:
}

We read with interest the recent editorial by CABIBBE et al. [1], which highlights the importance of rapid molecular tuberculosis (TB) assays for patient care.

Despite considerable advances over the last two decades, diagnosing TB in children remains challenging, even in high-resource settings. A recent publication reported the results of a large study conducted in eight countries that evaluated the performance of the Xpert MTB/RIF Ultra assay (Cepheid, Sunnyvale, CA, USA), a new molecular assay for the diagnosis of active TB [2]. Encouragingly, the authors reported that the Xpert MTB/RIF Ultra assay had higher sensitivity than the previous generation Xpert MTB/RIF assay $(63 \%$ versus $46 \%)$ in patients with sputum smear-negative, culture-positive pulmonary TB. Although no children and adolescents were included in this study, this finding has particular relevance for specialists providing clinical care for those age groups. A smaller, more recently published study using stored paediatric sputum samples provided further encouraging data [3].

Pulmonary TB in children is generally paucibacillary, and consequently most children have negative sputum-smear tests [4]. Although the first report on the Xpert MTB/RIF assay in children with pulmonary TB estimated its sensitivity to be $75.9 \%$ compared with culture [5], subsequent meta-analyses have shown its sensitivity to be substantially lower in this patient population $[6,7]$.

Prompted by the recent publication on the Xpert MTB/RIF Ultra assay, we conducted a survey among members of the Paediatric Tuberculosis Network European Trials Group (ptbnet), which currently includes 183 clinicians, epidemiologists and researchers based in 29 European countries [8, 9]. During a 2-week period in December 2017, all European members currently working clinically with paediatric patients were invited via the ptbnet online forum to participate in a web-based survey aiming to determine the current use of molecular TB assays. The participants knew they were participating in research and agreed to publication of the data they provided. According to current UK National Research Ethics Service regulations, research ethics committee review is not required for research involving healthcare staff recruited as research participants by virtue of their professional role (Governance Arrangements for Research Ethics Committees, paragraph 2.3.13).

A total of 91 ptbnet members, based at 72 healthcare institutions in 25 European countries (complete list at https://doi.org/10.5258/SOTON/D0487), participated in the survey. The majority of the participants were paediatric infectious diseases specialists $(n=55 ; 60.4 \%)$ or paediatric pulmonologists $(n=24 ; 26.4 \%)$, and were working at tertiary or quaternary healthcare centres $(n=81 ; 89.0 \%)$. All participants stated that they were currently providing care for children with TB and that they had access to molecular TB assays. At most healthcare institutions, Xpert MTB/RIF was used for routine clinical care $(n=60 ; 83.3 \%)$; at fewer institutions $(\mathrm{n}=11 ; 15.3 \%$ ) other commercial assays (GenoType MTBDRplus (Hain Lifescience GmbH, Nehren, Germany) $\mathrm{n}=8$; COBAS TaqMan MTB test (Roche Molecular Diagnostics, Pleasanton, CA, USA) $\mathrm{n}=3)$ or an in-house/non-commercial assay $(\mathrm{n}=13 ; 18.1 \%)$ were used. Only four $(5.6 \%)$ centres, located in Ljubljana (Slovenia), Sevilla (Spain), Vienna (Austria) and Zagreb (Croatia), had access to Xpert MTB/RIF Ultra for routine clinical care. At $16(22.2 \%)$ centres, two different molecular assays were available simultaneously.

@ERSpublications

Only few paediatric TB experts based in Europe had access to the Xpert MTB/RIF Ultra assay, a new generation molecular TB test, by the end of $2017 \mathrm{http}: / /$ ow.ly/ZgoF30jHShY

Cite this article as: Villanueva $\mathrm{P}$, Neth $\mathrm{O}$, Ritz N, et al. Use of Xpert MTB/RIF Ultra assays among paediatric tuberculosis experts in Europe. Eur Respir J 2018; 51: 1800346 [https://doi.org/10.1183/ 13993003.00346-2018]. 


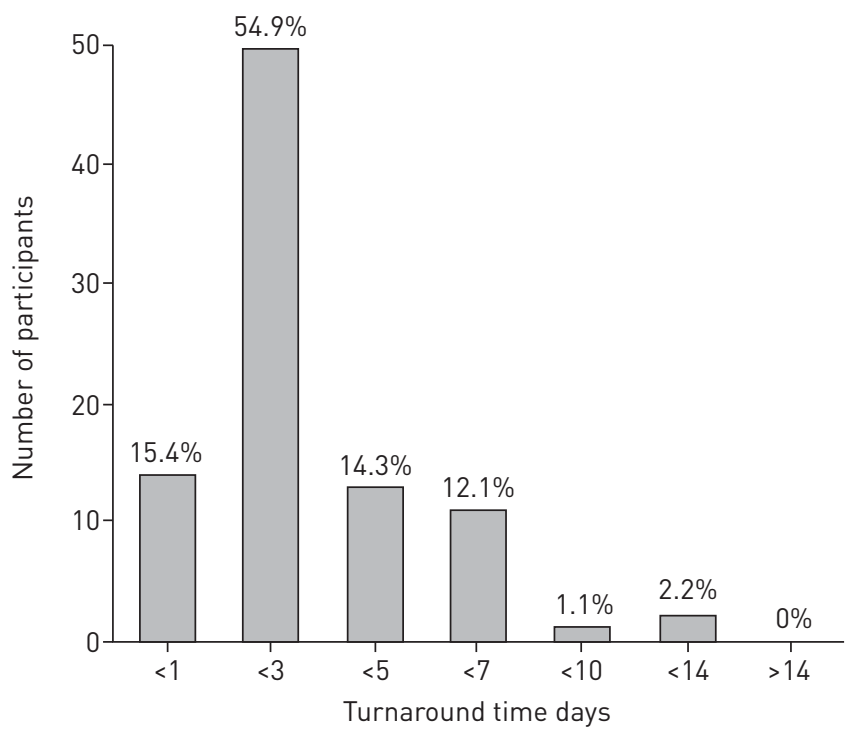

FIGURE 1 Summary of survey participants' responses $(n=91)$ regarding the average turnaround time (i.e. time between sending a sample and receiving a result) for molecular/PCR-based tuberculosis assays at their healthcare institution.

A large proportion of participants stated that they were routinely using PCR-based tests in children investigated for pulmonary TB to analyse the following samples: sputum ( $\mathrm{n}=85 ; 93.4 \%)$, gastric aspirates $(\mathrm{n}=79 ; 86.8 \%)$ and bronchoalveolar lavage samples $(\mathrm{n}=72 ; 79.1 \%)$; few participants were routinely testing stool samples by PCR in those patients $(n=11 ; 12.1 \%)$. Most participants stated that they were routinely performing PCR-based testing on a total of three samples in patients investigated for pulmonary TB $(n=47 ; 51.6 \%)$; fewer stated that they were routinely testing more than three $(n=1 ; 1.1 \%)$, two $(n=9 ; 9.9 \%)$ or one $(n=26 ; 28.6 \%)$ sample(s). Only eight $(8.8 \%)$ participants stated that they were not routinely performing PCR in this setting. Figure 1 shows the participants' responses regarding the turnaround time for molecular assays at their healthcare institution.

Furthermore, many participants stated that they were routinely using PCR-based assays to test lymph node tissue in patients with suspected TB lymphadenitis $(n=73 ; 80.2 \%)$, bone biopsy material in osteoarticular TB $(\mathrm{n}=56 ; 61.5 \%)$, cerebrospinal fluid in TB meningitis $(\mathrm{n}=73 ; 80.2 \%)$ and blood in miliary TB $(\mathrm{n}=37 ; 40.6 \%)$.

The survey results provide several interesting insights. First, despite the Xpert MTB/RIF Ultra assay having been launched in Europe in August 2017 and currently being available in 28 European countries (David Persing, Cepheid, Sunnyvale, CA, USA; personal communication), only a small minority of surveyed paediatric TB experts across Europe had access to this assay by the end of 2017. This is disappointing in light of the results of the aforementioned studies $[2,3]$, which indicate that patients, particularly with paucibacillary disease, would benefit from this new assay. Secondly, the results highlight that due to microbiological investigations having far lower yields in children than in adults [4], paediatric TB experts use molecular assays extensively, often testing multiple samples per patient. This approach is supported by several studies showing that analysis of multiple samples significantly increases the diagnostic yield in children $[5,10]$.

Potential limitations of our study include the limited sample size and the fact that survey participants mainly comprised research-active paediatric TB specialists, which may have introduced selection bias.

While the recent data on the Xpert MTB/RIF Ultra assay are encouraging, robust prospective studies in children will be needed to determine whether this assay can improve the diagnosis of TB in paediatric patients, who are often neglected in the evaluation of new diagnostic tools.

Paola Villanueva ${ }^{1}$, Olaf Neth $^{2}$, Nicole Ritz ${ }^{3,4}$ and Marc Tebruegge ${ }^{1,4,5}$ on behalf of the Paediatric Tuberculosis Network European Trials Group

${ }^{1}$ Dept of Paediatric Infectious Diseases and Immunology, Evelina London Children's Hospital, Guy's and St Thomas' NHS Foundation Trust, London, UK. ${ }^{2}$ Paediatric Infectious Diseases, Rheumatology and Immunology Unit, Hospital Universitario Virgen del Rocío, Instituto de Biomedicina de Sevilla (IBiS), Sevilla, Spain. ${ }^{3}$ University of Basel Children's Hospital, Paediatric Infectious Disease and Vaccinology, Paediatric Pharmacology and Pharmacometrics, and Migrant Health Service, Basel, Switzerland. ${ }^{4}$ Dept of Paediatrics, Royal Children's Hospital Melbourne, University of Melbourne, Melbourne, Australia. ${ }^{5}$ Dept of Infection, Immunity and Inflammation, UCL Great Ormond Street Institute of Child Health, University College London, London, UK. 
Correspondence: Marc Tebruegge, Evelina London Children's Hospital, Guy's and St Thomas' NHS Foundation Trust, 1 Lambeth Palace Road, London, SE1 7EU, UK. E-mail: marc.tebruegge@gstt.nhs.uk

Received: Feb 172018 | Accepted after revision: April 182018

Conflict of interest: M. Tebruegge reports non-financial support from Cepheid, as he is a co-investigator of a study that will receive support from Cepheid (laboratory materials). The manufacturer was not involved in any aspect of the present study, or the decision to submit the manuscript for publication.

\section{References}

1 Cabibbe AM, Sotgiu G, Izco S, et al. Genotypic and phenotypic M. tuberculosis resistance: guiding clinicians to prescribe the correct regimens. Eur Respir J 2017; 50: 1702292.

2 Dorman SE, Schumacher SG, Alland D, et al. Xpert MTB/RIF Ultra for detection of Mycobacterium tuberculosis and rifampicin resistance: a prospective multicentre diagnostic accuracy study. Lancet Infect Dis 2018; 18: 76-84.

3 Nicol MP, Workman L, Prins M, et al. Accuracy of Xpert MTB/RIF Ultra for the diagnosis of pulmonary tuberculosis in children. Pediatr Infect Dis J 2018; in press [https://doi.org/10.1097/INF.0000000000001960].

4 Tebruegge M, Ritz N, Curtis N, et al. Diagnostic tests for childhood tuberculosis: past imperfect, present tense and future perfect? Pediatr Infect Dis J 2015; 34: 1014-1019.

5 Nicol MP, Workman L, Isaacs W, et al. Accuracy of the Xpert MTB/RIF test for the diagnosis of pulmonary tuberculosis in children admitted to hospital in Cape Town, South Africa: a descriptive study. Lancet Infect Dis 2011; 11: 819-824.

6 Detjen AK, DiNardo AR, Leyden J, et al. Xpert MTB/RIF assay for the diagnosis of pulmonary tuberculosis in children: a systematic review and meta-analysis. Lancet Respir Med 2015; 3: 451-461.

7 World Health Organization. Automated real-time nucleic acid amplification technology for rapid and simultaneous detection of tuberculosis and rifampicin resistance: Xpert MTB/RIF assay for the diagnosis of pulmonary and extrapulmonary TB in adults and children. WHO/HTM/TB/2013.16. Geneva, World Health Organization, 2013. http://apps.who.int/iris/bitstream/10665/112472/1/9789241506335_eng.pdf

8 Tebruegge M, Ritz N, Koetz K, et al. Availability and use of molecular microbiological and immunological tests for the diagnosis of tuberculosis in Europe. PLoS One 2014; 9: e99129.

9 Ritz N, Brinkmann F, Santiago Garcia B, et al. Tuberculosis in young refugees. Lancet 2015; 386: 2475-2476.

10 Zar HJ, Workman L, Isaacs W, et al. Rapid molecular diagnosis of pulmonary tuberculosis in children using nasopharyngeal specimens. Clin Infect Dis 2012; 55: 1088-1095. 